\title{
Juvenile Ossifying Fibroma: Multiportal Endoscopic Trans-Nasal and Orbital Surgical Approaches, Histopathological and Radiological Findings
}

\author{
Tawfik Abdelaty Elkholy ${ }^{1, *}$ MD, Rehab Moustafa kamel ${ }^{2}$ MD, Bothina Ahmed Mohamed ${ }^{1}$ MD, Amany \\ Ibrahem Abosaif ${ }^{3}$ MD, Rania Rifat Abdel Maqsoud ${ }^{3}$ MD, Heba Mohamed Galal ${ }^{4}$ MD
}

\author{
* Corresponding Author: \\ Tawfik Abdelaty Elkholy \\ tawfikalkholy@gmail.com
}

Received for publication May 02 , 2021; Accepted June 29, 2021; Published online June 29, 2021.

Copyright The Authors published by Al-Azhar University, Faculty of Medicine, Cairo, Egypt. Users have the right to read, download, copy, distribute, print, search, or link to the full texts of articles under the following conditions: Creative Commons Attribution-Share Alike 4.0 International Public License (CC BY-SA 4.0).

doi: 10.21608/aimj.2021.73978.1464

\section{${ }^{1}$ Department}

Otorhinolaryngology, Al-Zahraa University Hospital, Faculty of Medicine for Girls, Al-Azhar University, Cairo, Egypt.

${ }^{2}$ Department of Ophthalmology, AlZahraa University Hospital, Faculty of Medicine for Girls, Al-Azhar University, Cairo, Egypt.

${ }^{3}$ Department of Pathology, Faculty of Medicine for Girls, Al-Azhar University, Cairo, Egypt.

${ }^{4}$ Department of Radiodiagnosis, AlZahraa University Hospital, Faculty of Medicine for Girls, Al-Azhar University, Cairo, Egypt.

\begin{abstract}
Background: Ossifying fibroma in the nasal cavity and paranasal sinus is a benign tumor histologically, however clinically may show invasive characteristics. It usually invades the orbital bone, the base of the skull and calvarium to induce orbital and cranial manifestations. It has malignancy rate of $0.4-0.5 \%$.

Aim of the study: to present two cases of Juvenile ossifying fibroma of the paranasal sinuses with orbital involvement, with special consideration to the complex surgical approaches and documenting the radiological finding and histopathological entities.

Patients and Methods: This study included two female children with Juvenile ossifying fibroma of the paranasal sinuses with orbital involvement. Research has been approved by the Research Ethical Committee of otolaryngology department, faculty of medicine for girls, AL -Azhar University. Both patients subjected to complete ENT and ophthalmological examination. CT study of the paranasal sinuses, orbit and brain were done using The two patients underwent excisional biopsy of the lesions through combined trans- nasal endoscopic and orbital approaches with repair of the orbital wall with titanium as in first case and/or high-density porous polyethylene sheets as in second case.

Post-operative follow up included complete ENT and ophthalmological examination and CT scan. The radiological findings and histopathogical entities of both cases were described in details.

Results: Both patients similarly presented with marked Swelling and masses of the right maxillary and ethmoidal sinuses that invaded the orbit with varying degree of proptosis, dystopia and visual affection. Our patients ages were 10 and 16 years. A histopathological feature of the first case is consistent with diagnosis of trabecular variant of ossifying fibroma, while the second case is consistent with the diagnosis of psammomatoid variant of ossifying fibroma. Radilogically were partially defined expansile heterogeneous fibro-osseous mass with ground glass appearance occupying the right maxillary, ethmoidal sinuses, and the right nasal cavity and invading the orbit. No intraoperative or Post-operative complication.
\end{abstract}

Conclusion: An accurate Diagnosis of JOF is made by correlation of clinical, radiological, histopathological findings. A combined Trans- Nasal endoscopic and orbital approach is effective for excision.

Keywords: ossifying fibroma; juvenile aggressive ossifying fibroma; fibro-osseous lesion.

Disclosure: The authors have no financial interest to declare in relation to the content of this article.

The Article Processing Charge was paid for by the authors.

Authorship: All authors have a substantial contribution to the article.

\section{CASE PRESENTATION (CLINICAL PRESENTATION:}

This study included 2 female patients presented to ENT and ophthalmology outpatient department at AlZahraa university hospital between January 2018 and January 2020. Both patients were presented with right nasal obstruction, facial disfigurement with varying degree of proptosis, dystopia and visual affection. The age was 10 and 16 years and both subjected to complete ENT and ophthalmological examination.

Radiological investigations included CT scan of the paranasal sinuses; orbit and brain. We prepared for surgery and performed excision of the tumor through combined trans- nasal endoscopic and orbital approaches with repair of the orbital wall with titanium or porous polyethylene sheets. In case 1 . We made our effort to dissect and remove as much as of the lesion adequately with only minor part of the tumor at the cranio-orbital wall medially and posteriorly could not be removed to guard against complications for the vision and CSF. Patients are followed up post-operatively according to regular schedule by clinical examination and endoscopic examination to remove any debris or crusts and clear the nasal passages. CT repeated once 6 months postoperatively.

Case-1: A 10-years-old female was presented with a progressively enlarging mass of her right cheek for the last 2 years and unilateral nasal obstruction on the right side of gradual onset for the last 5 months. Parents also noticed her right eye to have protruded (proptosis) since a year. Ocular examination disclosed a visual acuity of 6/6 (0.9) in the right and the left eyes. The intraocular pressure was 16 in both 
eyes by air buff tonometry. There was moderate proptosis, with Right horizontal lateral dystopia in the RT eye (Fig. 1). There was a slight RT exotropia. Hertel exophthalmometry revealed $4 \mathrm{~mm}$ right proptosis, the expanded lamina paprycea and nasal bone can be palpated. Fundus examination revealed no indentation of the globe by an orbital mass and no choroidal fold.

A CT of the head with specific protocol requested by ophthalmologist revealed a right paranasal sinus Lesion involving the right orbit and was subsequently referred to the Department of Otolaryngology for Consultation.

Nasal endoscopy after decongestion revealed a mass obstructing the nasal cavity above the Inferior turbinate and pushing the septum off the middle line towards the left side. The mass looked pink with smooth surface, by fine suction it was firm, not compressible and obstructing the right nasal cavity completely, the Middle turbinate could not be identified or demarcated from the mass.

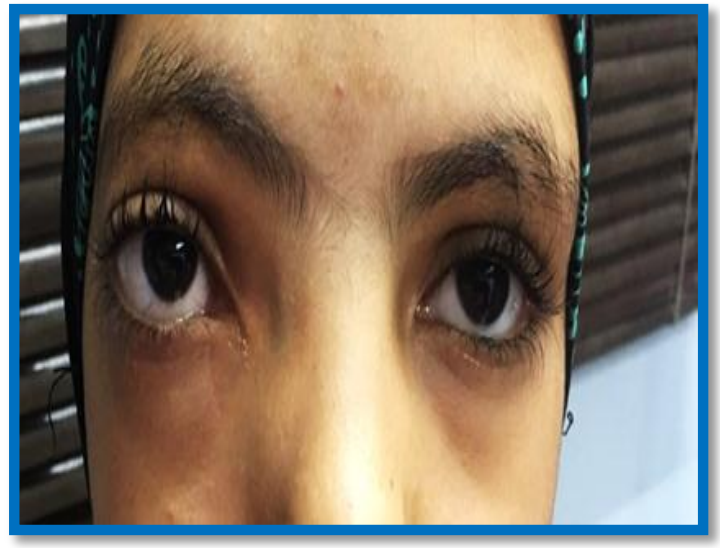

Fig. 1: Showing Patient 1 at presentation with dystopia, proptosis and ocular deviation (Notice the Inner Canthus widening with increased distance between nasal bridge and MPL on the right side).

Review of the CT scan of the orbit, brain and paranasal sinuses (fig. 2 a, b) revealed a partially defined expansible heterogeneous fibro-osseous mass occupying the right ethmoid and right nasal cavity measuring about $48 \times 35 \times 4 \mathrm{~mm}$ in diameters surrounded by smooth well-defined cortical bone shown as ground glass appearance, surrounded by smooth well-defined cortical bone the mass extended into the right maxillary sinus with obliteration of ostiomeatal complex and complete opacification of right maxillary, ethmoid and sphenoid sinuses. It caused displacement of the medial and inferior orbital plates with rarefaction of the bony margins and displacement of the right globe laterally with proptosis. There is also displacement and rarefaction of the medial wall of the right maxillary sinus and the orbital floor inferiorly.

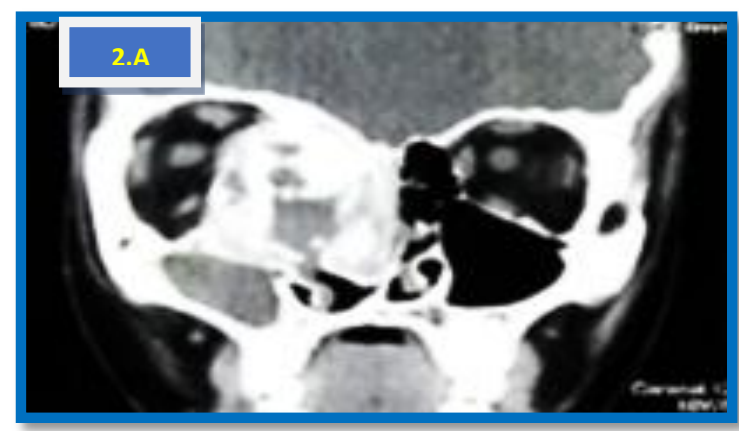

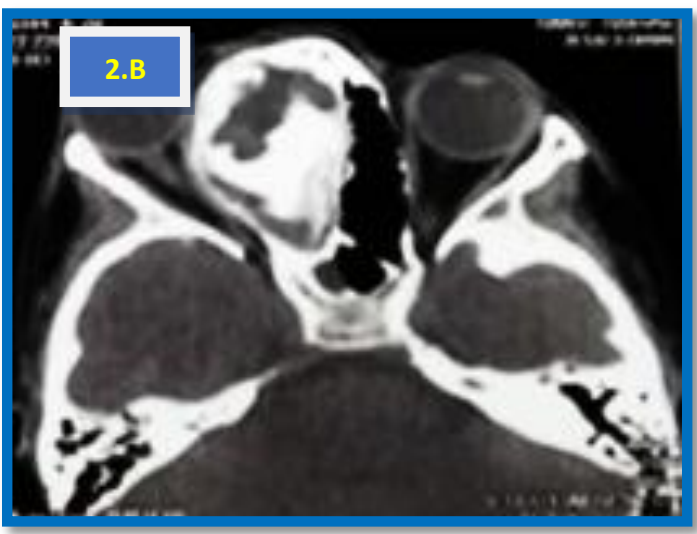

Fig. 2: Non contrast $C T$ scan paranasal sinus reformatted coronal view (A), axial view (B).

Surgical technique: Planning and designing of surgical repair

We reviewed the radiological data of the patient, which obtained by using Thin-section axial CT scans of $0.625-1 \mathrm{~mm}$ thickness contiguous slice section under bone window settings. Then multiplanar reformation was performed and 3D images were reconstructed.

CT data were processed and transferred in a digital image via DICOM files to a compact disc (CD) on (CD)-Rom. Dicom file of the axial cuts were imported to the surgical planning software (MIMICS, Materialise, Leuven, Belgium) for data conditioning and image processing. The steps of computer assisted surgical planning based on CT data were performed by MIMICS program.

A new 3D virtual model was rebuilt where all the air spaces surrounding the unaffected orbit in the virtual model were filled in. Virtual correction was made by creating a mirror image. Cutting through 3D image was performed to the mirrored orbital side followed by splitting and separating different cutting planes were used to reach the selected area, which wanted for stereo lithographic template prototyping.

Anatomical orbital model was built, as the virtual model data was converted to STL format and solid physical models were created from acrylic resin, by rapid prototyping machine using a $3 \mathrm{D}$ printer.

Under GA with modified Trendelenburg position and hypotensive anesthesia technique, the nasal passage was prepared by Xylometazoline soaked cotton patties, placed for 5-7 minutes, then endoscope passed to outline and reassess the mass, and then we injected the surroundings of the mass intra-nasally and spheno-palatine fossa trans-orally through greater palatine foramen with Xylocaine-Epinephrine $1 / 100000$ concentration.

We tried to identify the middle turbinate and manipulate the underlying mass to find out dissection plane, the main part was arising from the maxillary sinus and filling the nasal cavity up to ethmoidal region. 
During endoscopic nasal excision the mass in the maxillary sinus is followed up and dissected to the ethmoidal region where we encountered a deficient lamina papyrecia, so fovea ethmoidalis seems to be violated by the tumor and also the orbital floor was invaded as confirmed by the preoperative CT. The tumor was partially capsulated and could be excised only as piecemeal with intact ethmoid roof.

In collaboration with Ophthalmology surgeons, we proceeded with Transcrancular Transconjunctival incisions with lateral canthotomy and inferior cantholysis to approach the tumor from the orbital side. We disregarded extended lynch insion for better cosmesis. Transcrancular incision was made Lateral to the caruncle, using Westcott scissors transconjunctival incision was made by a blunttipped scissors to dissect in the subconjunctival plane just below the tarsal plate, blunt dissection was continued to expose the orbital part of the tumor.

After excision of the mass the bony defect of the medial wall and floor of the orbit was repaired by the preoperatively designed a $3 \mathrm{~d}$ demo for the right orbit titanium mesh $(0.3-\mathrm{mm}$ original semi rigid titanium mesh (Stryker- Leibinger).UK Ltd) bent, adjusted and fitted on the preoperatively computer assisted, custom made template and by porous polyethylene sheet (micro thin sheet 0.45 omnipore matrix surgical USA), Fig (3), Fig (4).

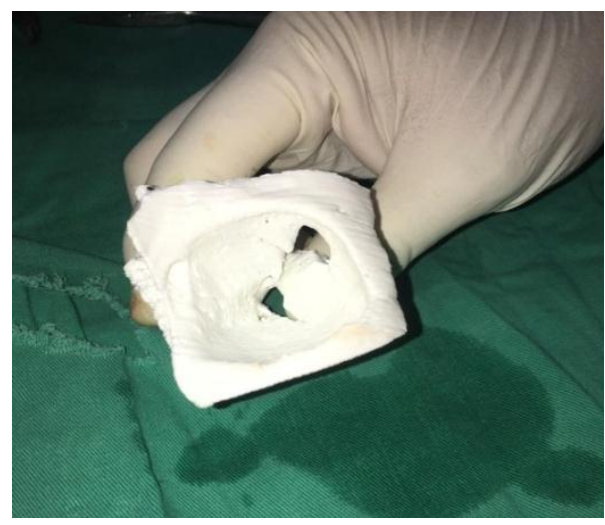

Fig. 3: showing the implant material used for reconstruction.

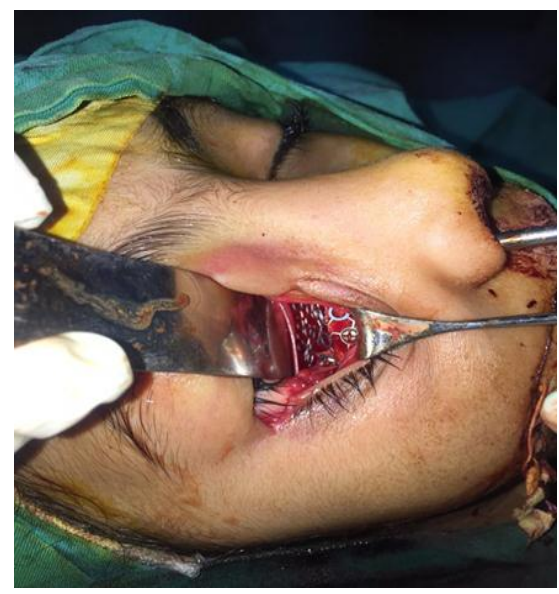

Fig. 4: showing the procedure with titanium sheet implants in place to reconstruct the defect.
Post-operative photos showed marked improvement of dystopia and ocular deviation (Fig (5 a, b)

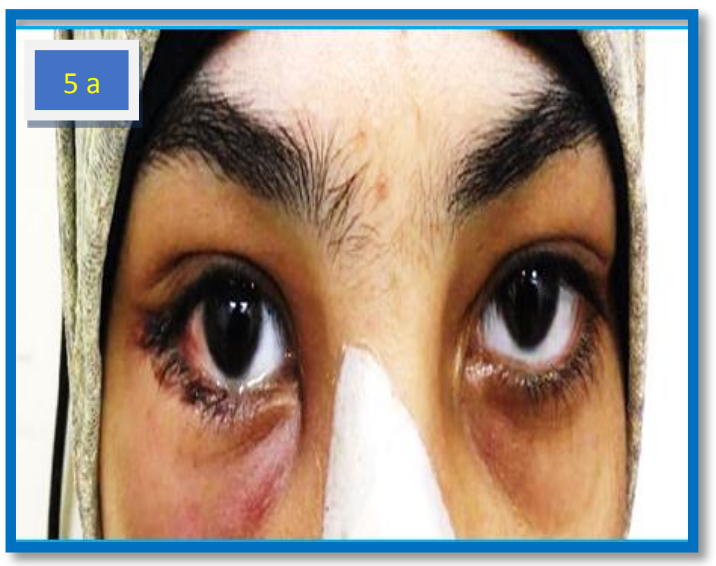

Fig 5: (a, b) Marked improvement of dystopia and

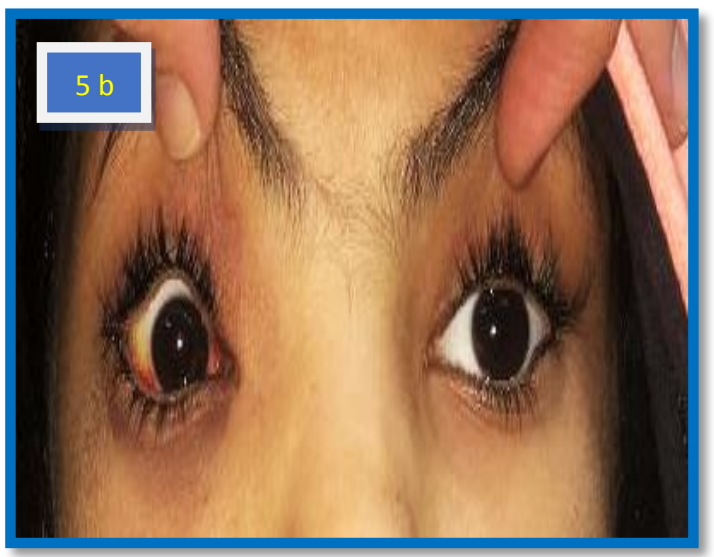

ocular deviation Post-operatively.

Post-operative CT scans revealed subtotal excision of the mass with adequate reconstruction of the orbital wall.

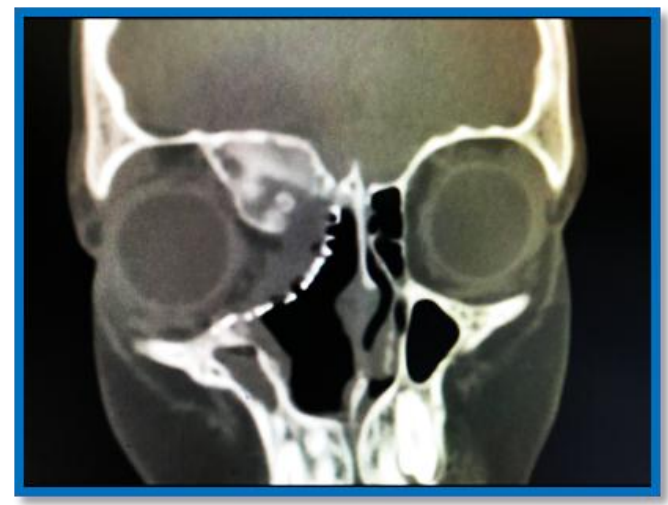

Fig 6: coronal view bone window showing: subtotal excision of the mass.

Histopathologic examination of the specimen showed multiple fragments of rubbery grayish tan tissue with firm to hard areas collectively measured $10 \times 10 \mathrm{~cm}$ (Fig.7). 


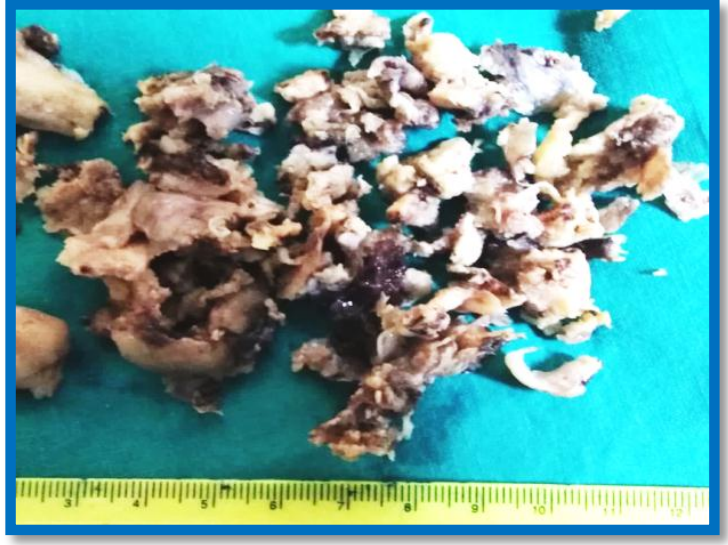

Fig 7: Multiple tan-white fragments, with rubbery to hard, firm to gritty cut section.

Tissues prepared and stained with hematoxylin and eosin. Microscopic examination revealed bony trabeculae surrounded by plump osteoblasts. The bony trabeculae embedded in cellular fibrous stroma (Fig: 8.) and this was consistent with the trabecular variant of ossifying fibroma.

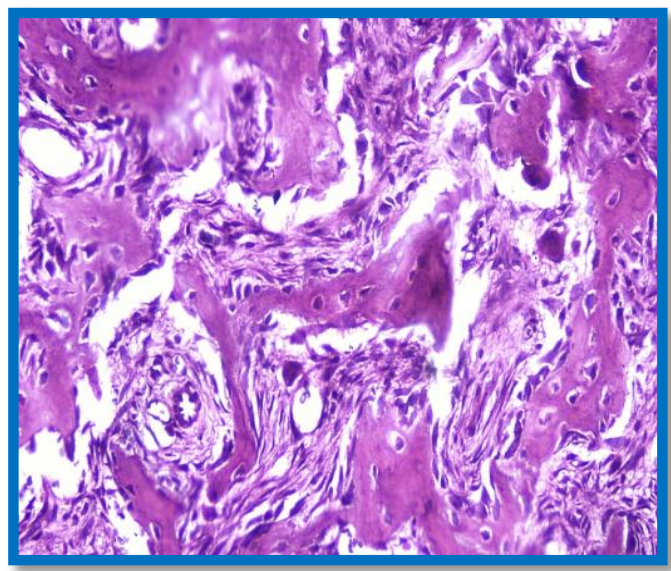

Fig 8: Bony trabeculae with rim of plump osteoblastic cells embedded in cellular fibroblastic stroma (Hematoxylin and eosin stain: Magnification power 20x.)

Case-2: patient was a 16- years-old female presented with a progressively upward dystopia of the right eye for the last 1 year with right sided nasal obstruction. Ocular examination disclosed a visual acuity of $6 / 6$ (0.9). The intraocular pressure was 16 for the Right eye and 18 for the left air buff tonometry. There was Right upward dystopia (Fig. 9). Free EOM (extraocular muscles) in the 6 cardinal directions. Fundus examination revealed no indentation of the globe by any orbital mass and no choroidal fold.

Nasal endoscopy revealed pale blue mucosa of the nasal cavity with no remarkable septal deviation with a large right hard Nasal mass, pinkish in color with smooth surface, with probing we could only passed inferior to the mass in very tiny space over the nasal floor.
The patient gave a history of incisional biopsy of the mass with histopathological report of fibro-osseous lesion.

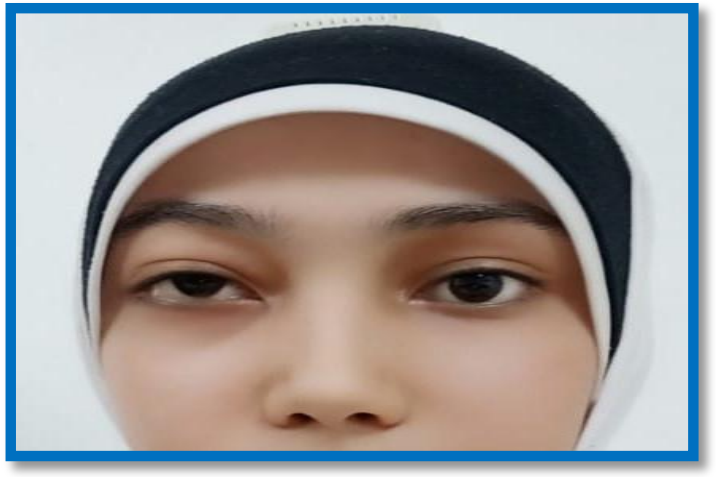

Fig. 9: Patient 2 showed dystopia and ocular deviation preoperatively.

CT of the patient revealed a partially defined expansible heterogeneous fibro-osseous mass with ground glass appearance occupying the right maxillary sinus measuring about $5 \times 4 \mathrm{~cm}$. Bony erosion of medial as well as lateral and superior maxillary sinus walls with encroachment upon the right nasal cavity and right orbital floor while the nasal septum was not remarkably deviated (Fig 10 ).

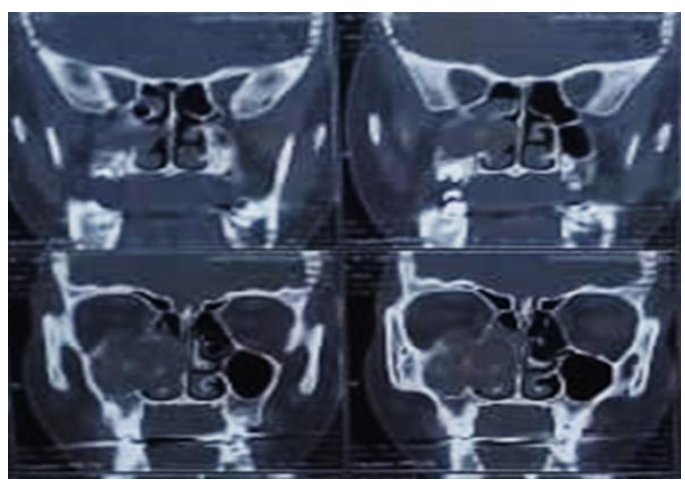

Fig. 10: Non contrast CT scan of the paranasal sinuses, coronal views

So we tried to achieve total excision and repair the defect of the orbital floor by porous polyethelene. However; by endoscopic approach, the nasal portion could only be removed in a piecemeal while orbital portion could be dissected and removed in-toto as single capsulated mass, (Fig 11), (Fig 12)

During dissection of intra-orbital part when nearby the optic nerve, the eye observed by ophthalmologist tell me stop the procedure if mydriasis occur and recur on myosis after minutes. 


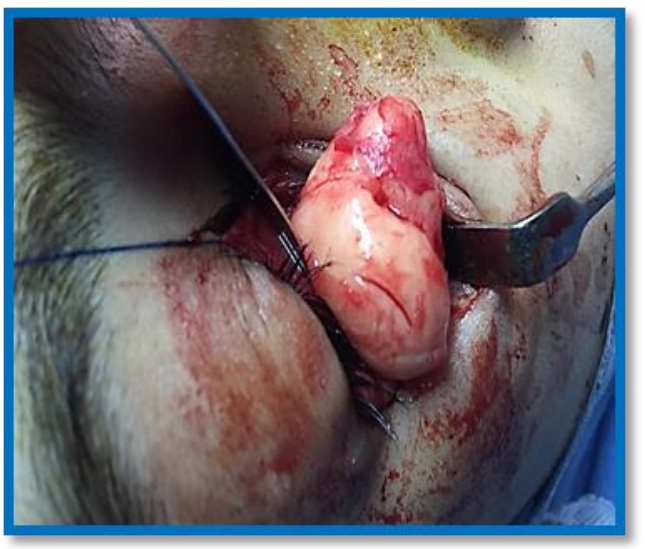

Fig. 11: intraoperative view showing delivery of the orbital part of the mass after careful dissection and release from surrounding tissues.

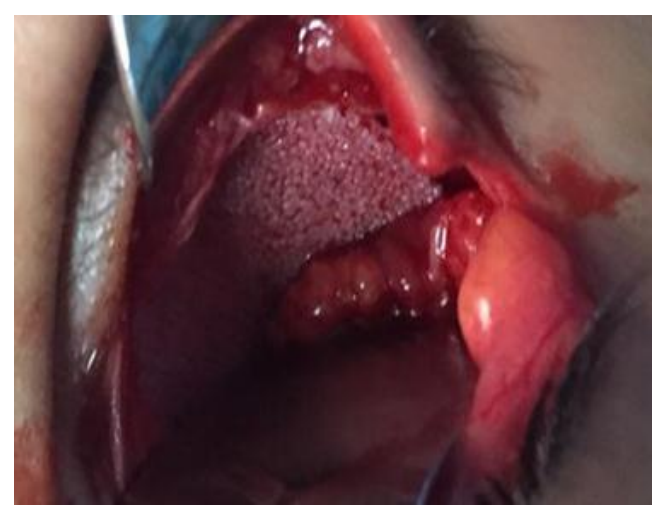

Fig. 12: Repair of the medial orbital wall using highdensity porous polyethylene sheets.

Histopathological examination of removed tissues revealed well circumscribed pink mass with smooth outer surface measuring $3 \times 2 \mathrm{~cm}$ and multiple tan to brown fragments of tissue collectively measured $5 \times 4 \mathrm{~cm}$. Cut section showed firm white tissue with bony fragments.

Microscopic examination of the specimen showed cellular fibrous stroma with small bony ossicles with many psammoma bodies. A small focus of bone trabeculae lined by plump osteoblastic cells (Fig: 13) and this is consistent with the diagnosis of psammomatoid variant of ossifying fibroma.

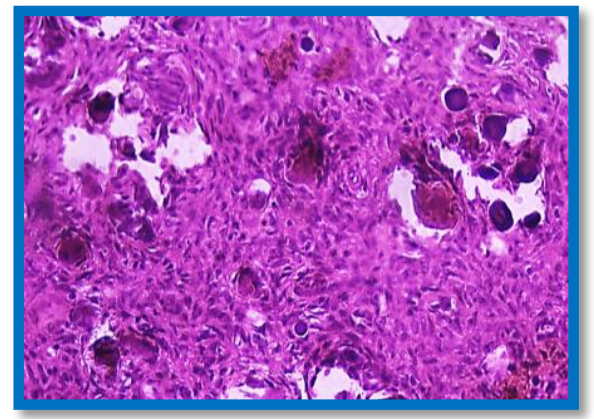

Fig. 13: Small bony ossicles within cellular plump fibroblastic stroma and some psammoma bodies (magnification power 20x).
Post-operative non contrast CT scan of paranasal sinus showed subtotal excision of the mass (Fig; 14).

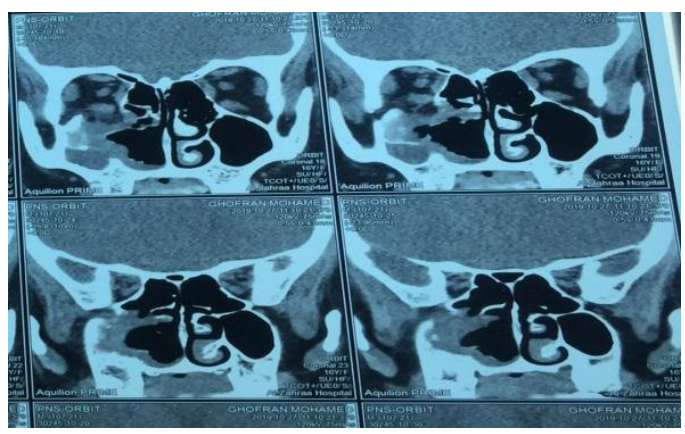

Fig. 14: coronal view bone window showing: subtotal excision of the mass.

\section{DISCUSSION}

Fibro-osseous benign lesions are not common in the sinonasal region. Histopathologically 3 entities have been described, namely osteoma, fibrous dysplasia and ossifying fibroma . ${ }^{1}$ However they share several clinical, radiological and histological Features with variable behaviors.

Ossifying fibroma (OF) is a rare benign fibroosseous lesion which was first described by Menzel in 1872. He considered it as a form of Osteoma.1, 2 but the term "Ossifying Fibroma" was subsequently coined by Montgomery in $1927.2,3$

Odontogenic, developmental and traumatic origins have been suggested but no definite etiology have been approved. Maxillary ossifying fibroma is uncommon and orbit is rarely affected. ${ }^{4}$

In head and neck region the mandible is affected in about $62 \%$ to $89 \%$ of patients 7 followed by the maxilla and rarely the orbit, skull base and calvarium. $^{5}$

While ossifying fibroma of the sinonasal tract occurs at a slightly older age (3rd to 4th decade of life); we presented 2 cases of Ossifying Fibroma in younger population, 10 and 16 years (Juvenile OF) as far as we know this is rarely reported in literature.

Involvement of the sinonasal tract is extremely rare and only 48 cases were reported in the literature from 1971 to 2011 on the basis of a search in PubMed. The reported cases were between 3 weeks and 41 years of age, and $23.7 \%$ to $56 \%$ were female. $^{6}$

Extra mandibular lesions such as those occur in the paranasal sinuses and midface tends to display more aggressive behaviour and rapid growth ${ }^{7}$

We presented two cases of Ossifying fibroma with involvement of Sino-orbital region, maxillary and ethmoidal sinuses which is considered rare as per (according to) literature review.

Our Case reports comprises two female children; review of literatures showed that Fibro-osseous lesions tend to be more common in females.$^{8}$ some authors ${ }^{9}$ found female predominance to be $69 \%$ but 
some studies reported equal affection for both genders.${ }^{10}$ Our patients were 10 and 16 years old; however Ossifying fibroma of the sinonasal tract occurs at a slightly older age (3rd to 4th decade of life, and preferentially in black women). So we reported both cases as juvenile Ossifying fibroma in contrast to literature and case report review.

Both patients presented with ocular symptoms with varying degrees of dystopia, proptosis and nasal obstruction which is the most common presentation as described in case reports and literature. Clinically, the presenting symptoms of ossifying fibroma are variable and are dependent on the location of the tumor causing mass effect such as nasal obstruction, headache and rhinorrhea. Larger tumors involving the midfacial region can lead to facial deformity. Ocular symptoms comprises epiphora, proptosis, diplopia, and decreased visual acuity when the orbit is invaded It may presents as painless swelling with rapid growth mimicking malignancy such as osteosarcoma. ${ }^{11-14,16}$

Radiological findings in both cases revealed a partially defined expansile heterogeneous fibroosseous mass with ground glass appearance occupying the right maxillary and ethmoidal sinuses and invading the right orbit which is the typical descriptive radiology of such lesions with little variability between cases according to fibrous and osseous components percentage . ${ }^{15}$ The radiographic presentations of our patients were consistent with the features of juvenile ossifying fibroma with respect to rapidly progressive nature, and the well-delineated radiographic picture.

Histologically there are two variants of juvenile OF; trabecular and psammomatous types. The trabecular variant is composed of a fibroblastic spindle cell stroma, containing bands of cellular osteoid without osteoblastic rimming together with slender trabeculae of immature bone with a rim of enlarged osteoblasts. Sometimes these trabeculae may anastomose to form a lattice. Scattered clusters of multinucleated giant cells; mitoses may be present.$^{17}$

The juvenile psammomatoid variant (JPOF) is characterized by a proliferation of benign fibroblastic stroma containing small spherical mineralized material (ossicles) having vague concentric lamination resembling psammoma bodies.$^{15}$ The ossicles themselves may also fuse to form trabeculae showing reversal lines. Other features such as trabeculae of woven bone as well as lamellar bone, pseudocystic stromal degeneration and haemorrhages, multinucleate giant cells, and normal mitotic figures may be present. JPOF has to be distinguished from extracranial meningioma with psammoma bodies, which demonstrates EMA positivity. Moreover, the psammomatoid ossicles in JPOF are clearly different from the acellular spherical true psammoma bodies . ${ }^{14,18,19}$

JPOF may be confused with fibrous dysplasia. The most important distinguishing features are the rapid rate of growth, presence of well delineated radiographic margin as well as in appearances of mineralized material serves to distinguish OF from fibrous dysplasia.$^{2}$

Distinction between Ossifying Fibroma and osseous dysplasia on histologic grounds only may be problematic as both entities share the variation in stromal cellularity and appearances of mineralized material. Clinical presentation and radiographic appearance may be decisive .,20 $^{2,0}$

In the current study case- 1 histological features was consistent with trabecular variant while case- 2 was consistent with psammomatoid variant.

\section{CONCLUSION}

An accurate diagnosis of JOF could be made by correlation of clinical, radiological, histopathological finding. Combined Trans- Nasal endoscopic and orbital approaches with repair of the orbital wall with titanium or porous polyethylene sheets are effective surgery for aggressive JOF.

\section{Consent}

Informed written consent was obtained from patient's parents and also in accordance to the guidelines of the local ethics committee of Al-Azhar University.

\section{REFERENCES}

1- Cliniglioappiani M, Verillaud B, BressonD: Ossifing Fibroma Of Paranasal Sinus: Diagnosis And Mangement.Acta Otorhinolaryngol Ital. 2015 Oct; 35(5): 355-361.

2- Slootweg PJ and El-Mofty SK. Ossifying fibroma. In: El-Naggar AK, Chan JKC, Grandis JR, Takata T, Slootweg PJ, editors. WHO Classification of Head and Neck Tumours. 4th. Lyon: IARC, 2017; 319-29.

3- J.C. Martins Junior, F.S. Keim and M.S. Kreibich. Peripheral ossifying fibroma of themaxilla: case report, Int. Arch. Otorhino-laryngol; 2008, 12: 295-9.

4- I. Baumann, R. Zimmermann, F. Dammann and M.M. Maassen. Ossifying fibroma ofthe ethmoid involving the orbit and the skull base, Otolaryngol Head NeckSurg; 2005, 133 (1) (2) 158-9

5- Speight PM and Takata T. New tumour entities in the 4th edition of the World Health Organization Classification of Head and Neck tumours: odontogenic and maxillofacial bone tumours. Virchows Arch. 2018, 472 (3):331-9. Doi: 10.1007/s00428-017-2182-3

6- Mohsenifar Z, Nouhi S Abbas FM, et al. Ossifying fibroma of the ethmoid sinus: report of a rare case and review of literature, J. Res. Med. Sci; 2011, 16 (6), 841-847.

7- Choi YC, Jeon EJ and Park YS. Ossifying fibroma arising in the right ethmoid sinus and nasal 
cavity, Int. J. Pediatr. Otorhinolaryngol; 2000, 54: (2-3), 159-162.

8-Etetafia M, Enemudo M and Omoregie F. Giant ossifying fibroma of the maxillary region: case series; 2019, 48(1): 92-103

9- El-Mofty SK, Nelson B and Toyosawa S. Ossifying fibroma. In: El-Naggar AK, Chan JKC, Grandis JR, Takata T, Slootweg PJ, editors WHO Classification of Head and Neck Tumours. 4th. Lyon: IARC; 2017, pp. 251-2.

10- Lemoine S, Cassagnau E, Bertin, et al. Juvenile ossifying fibroma: case report and literature review. Management and differential diagnosis. $J$ Oral Med Oral Surg ; 2018,24:67-71

11- Keles B, Duran M, Uyar Y, et al. Juvenile ossifying fibroma of the mandible: a case report. J Oral Maxillofac Res; 2010, 1(2):5. doi: 10.5037/jomr.1205.

12- Brannon RB \& Fowler CB. Benign fibro-osseous lesions: a review of current concepts. Adv Anat Pathol 2001, 8: 126-43.

13- El Mofty S. Psammomatoid and trabecular juvenile ossifying fibroma of the craniofacial skeleton: two distinct clinicopathologic entities. Oral Surg Oral Med Oral Pathol Oral Radiol Endod. 2002, 93: 296-304.

14- Manes RP, Ryan MW, Batra PS, et al. Ossifying fibroma of the nose and paranasal sinuses, Int. Forum Allergy Rhinol. 2013, 3(2): 161-8.
15- Rao S, Nandeesh BN, Arivazhagan A, et al. Psammomatoid juvenile ossifying fibroma: Report of three cases with a review of literature. $J$ Pediatr Neurosci; 2017, 12:363-6

16- Wang H, Sun X, Liu Q, et al. Endoscopic resection of sinonasal ossifying fibroma: 31 cases report at an institution. Eur Arch Otorhinolaryngol. 2014, 271:2975-82.

17- Bertrand B, Eloy P, Cornelis JP, et al. Juvenile aggressive cemento-ossifying fibroma: case report and review of the literature. Laryngoscope.1993, 103(12):1385-90.

18- Thompson LD \& Gyure KA. Extracranial sinonasal tract meningiomas: a clinicopathologic study of 30 cases with a review of the literature. Am J Surg Pathol. 2000, 24: 640-50

19- Slootweg PJ, Panders AK, Koopmans R, et al. Juvenile ossifying fibroma. An analysis of 33 cases with emphasis on histopathological aspects. J Oral Pathol Med.;1994, 23:385-8

20- Chatterjee A, Ajmera N and Singh A. Peripheral cemento-ossifying fibroma of maxilla, J. Indian Soc. Periodontal. 2010, (3):186-189. 